\title{
完全二部图 $\boldsymbol{K}_{m, n}$ 的 $\boldsymbol{K}_{p, q}$ 因子分解 ${ }^{*}$
}

\author{
杜北梁 \\ 王 建 \\ (苏州大学数学科学学院, 苏州 215006) \\ (南通职业大学, 南通 226007)
}

摘要 如果完全二部图 $K_{m, n}$ 的边集可以划分为 $K_{m, n}$ 的 $K_{p, q}$ - 因子, 则称 $K_{m, n}$ 存在 $K_{p, q^{-}}$因子分解. 给出 $K_{m, n}$ 存在 $K_{p, q^{-}}$因子分解的一个充分条件. 同时证 明: 对于任意正整数 $k$, 当 $p: q=k:(k+1)$ 时, $K_{m, n}$ 存在 $K_{p, q^{-}}$因子分解, 即 Martin 的 BAC 猜想成立.

\section{关键词 完全二部图 因子分解 $\mathrm{HUBMFS}_{2}$ 方案}

$K_{m, n}$ 表示完全二部图, 其两个部分点集 $X$ 和 $Y$ 分别具有 $m$ 和 $n$ 个点. 如 果 $K_{m, n}$ 的一个子图 $F$ 包含了 $K_{m, n}$ 的所有点, 则称 $F$ 为 $K_{m, n}$ 的支撑子图. 若 $K_{m, n}$ 的支撑子图 $F$ 的每个分支均同构于 $K_{p, q}$, 则称 $F$ 为 $K_{m, n}$ 的 $K_{p, q^{-}}$因子. 如果 $K_{m, n}$ 的边集可以划分为 $K_{m, n}$ 的 $K_{p, q}$ - 因子, 则称 $K_{m, n}$ 存在 $K_{p, q^{-}}$因子分 解. 在文献 [1] 中, 称 $K_{m, n}$ 的 $K_{p, q^{-}}$因子分解为可分解的 $(m, n, p+q, 1)$ 二部 $K_{p, q^{-}}$ 设计. 如果 $K_{m, n}$ 存在 $K_{p, q}$ - 因子分解, 则称 $K_{m, n}$ 是可 $K_{p, q}$ - 因子分解的. 本文 用到的图论方面的名词术语, 均参照文献 [2].

$K_{m, n}$ 的 $K_{p, q^{-}}$因子分解有许多应用, 特别是 Yamamoto 等 ${ }^{[3]}$ 用其建立了计 算机数据存储的 $\mathrm{HUBMFS}_{2}$ 方案. Ushio ${ }^{[4]}$ 完全解决了 $K_{m, n}$ 的 $K_{1,2}$ - 因子分解存 在性问题, 这是至今该问题 $q \neq p$ 情形下的惟一的一个完整结果. Wang ${ }^{[5]}$ 对 $q$ 是 素数的情形给出了 $K_{m, n}$ 存在 $K_{1, q}$ - 因子分解的一个充分条件. 随后，在将 Wang 的结论由 $q$ 为素数推广到 $q$ 为任意正整数方面又做了许多工作. 在文献 [6,7] 中, 本文的第一作者将 Wang 的结论推广到 $q$ 为素数幂和素数积的情形. 最近我们又 证明了 Wang 的结果对 $q$ 为任意正整数都成立 (参阅文献 [8] 的定理 1.4).

定理 $\mathbf{1}^{[8]}$ 设 $q$ 为任意正整数. 如果满足下述 4 个条件: (1) $m \leqslant q n,(2) n \leqslant$ $q m,(3) q m-n \equiv q n-m \equiv 0\left(\bmod \left(q^{2}-1\right)\right),(4)(q m-n)(q n-m) \equiv 0(\bmod q(q-$ 1) $\left.\left(q^{2}-1\right)(m+n)\right)$, 则 $K_{m, n}$ 存在 $K_{1, q^{-}}$因子分解.

*国家自然科学基金资助项目 (批准号: 10071056) 
Martin 研究了 $K_{m, n}$ 的 $K_{p, q^{-}}$因子分解, 给出 $K_{m, n}$ 存在 $K_{p, q^{-}}$因子分解的必 要条件 (文献 [9] 的定理 2.5).

定理 $2^{[9]}$ 设 $m, n, p, q$ 为正整数, $q \neq p$ 且 $p q>1$. 如果 $K_{m, n}$ 存在 $K_{p, q^{-}}$ 因子分解, 则下列各式都是整数:

$$
\begin{gathered}
\frac{m+n}{p+q}, \frac{p m-q n}{p^{2}-q^{2}}, \frac{p n-q m}{p^{2}-q^{2}}, \frac{m n(p+q)}{p q(m+n)}, \\
\frac{(p n-q m) n}{p(p-q)(m+n)}, \frac{(p m-q n) n}{q(p-q)(m+n)}, \frac{(p m-q n) m}{p(p-q)(m+n)}, \frac{(p n-q m) m}{q(p-q)(m+n)} .
\end{gathered}
$$

Martin 称定理 2 中的条件为 $K_{m, n}$ 存在 $K_{p, q}$ - 因子分解的基本算术条件, 简 称 BAC 条件. 并给出下面的猜想 (文献 [9] 的猜想 2.7).

BAC 猜想 ${ }^{[9]}$ 如果正整数 $m, n, p, q$ 满足 $\mathrm{BAC}$ 条件, 则 $K_{m, n}$ 存在 $K_{p, q^{-}}$ 因子分解.

本文将定理 1 的结论推广到 $K_{m, n}$ 存在 $K_{p, q^{-}}$因子分解情形: 对于正整数 $p, q(q>p)$, 给出 $K_{m, n}$ 存在 $K_{p, q^{-}}$因子分解的一个充分条件. 同时证明：对于任 意正整数 $k$, 当 $p: q=k:(k+1)$ 时, BAC 猜想成立. 即我们将证明如下结论:

定理 3 设 $p, q$ 为正整数, $q>p$. 如果满足下述 4 个条件: (1) $p n \leqslant q m,(2)$ $p m \leqslant q n,(3) q m-p n \equiv q n-p m \equiv 0\left(\bmod \left(q^{2}-p^{2}\right)\right),(4)(q m-p n)(q n-p m) \equiv$ $0\left(\bmod p q(q-p)\left(q^{2}-p^{2}\right)(m+n)\right)$, 则 $K_{m, n}$ 存在 $K_{p, q^{-}}$因子分解.

定理 4 对于任意正整数 $k$, 当 $p: q=k:(k+1)$ 时, BAC 猜想成立.

\section{1 定理 3 的证明}

定理 3 的证明由以下几个引理构成. 第 1 个引理是显然的, 其中 $\operatorname{gcd}(x, y)$ 表 示 $x$ 和 $y$ 的最大公约数.

引理 1 设 $u, v, x$ 和 $y$ 是正整数. 如果 $\operatorname{gcd}(u x, v y)=1$, 则 $\operatorname{gcd}(u v, u x+v y)=$ 1.

引理 2 设 $s$ 为任意正整数. 如果 $K_{m, n}$ 存在 $K_{p, q}$ - 因子分解, 则 $K_{s m, s n}$ 存 在 $K_{p, q^{-}}$因子分解.

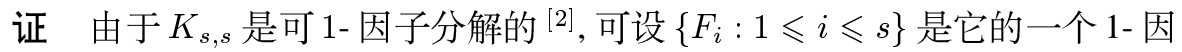
子分解. 对于每一个 $1 \leqslant i \leqslant s$, 用 $K_{m, n}$ 代替 $F_{i}$ 的每条边, 即得到 $K_{s m, s n}$ 的一 个支撑子图 $G_{i}$, 且 $G_{i}(1 \leqslant i \leqslant s)$ 边集的并为 $K_{s m, s n}$. 由于 $K_{m, n}$ 是可 $K_{p, q}$ - 因子 分解的, 因而 $G_{i}$ 也是可 $K_{p, q^{-}}$因子分解的. 所以 $K_{s m, s n}$ 存在 $K_{p, q^{-}}$因子分解.

由引理 2 可得

推论 对于任意正整数 $s, K_{s p, s q}$ 存在 $K_{p, q}$ - 因子分解.

由推论我们只需考虑 $p m<q n, p n<q m$ 的情形. 在这种情形下, 令

$a=\frac{q n-p m}{q^{2}-p^{2}}, b=\frac{q m-p n}{q^{2}-p^{2}}, r=\frac{(p+q) m n}{p q(m+n)}, c=\frac{(q m-p n)(q n-p m)}{p q(p+q)(m+n)}$.

由定理 3 的条件 (1) (4) 可知 $a, b, c$ 是整数, 且 $0<a<m, 0<b<n$.于 是有 $a p+b q=m, a q+b p=n$. 进而可得 $r=a+b+c$, 并且它也是整数. 设 
$\operatorname{gcd}(a p, b q)=d, a p=d e, b q=d k$, 其中 $\operatorname{gcd}(e, k)=1$. 因为 $c \equiv 0\left(\bmod (q-p)^{2}\right)$, 令 $z=c /(q-p)^{2}$, 即可得下列:

$$
\begin{gathered}
d=\frac{p q(q e+p k) z}{e k}, r=\frac{(e+k)\left(q^{2} e+p^{2} k\right) z}{e k}, m=\frac{p q(e+k)(q e+p k) z}{e k}, \\
n=\frac{\left(q^{2} e+p^{2} k\right)(q e+p k) z}{e k}, a=\frac{q e(q e+p k) z}{e k}, b=\frac{p k(q e+p k) z}{e k} .
\end{gathered}
$$

记 $q=q_{1}{ }^{k_{1}} q_{2}{ }^{k_{2}} \cdots q_{\gamma}{ }^{k_{\gamma}}$, 其中 $q_{1}, q_{2}, \cdots, q_{\gamma}$ 是两两互异的素数, $k_{1}, k_{2}, \cdots, k_{\gamma}$ 是正整 数; $p=p_{1}{ }^{h_{1}} p_{2}{ }^{h_{2}} \cdots p_{\omega}{ }^{h_{\omega}}$, 其中 $p_{1}, p_{2}, \cdots, p_{\omega}$ 是两两互异的素数, $h_{1}, h_{2}, \cdots, h_{\omega}$ 是正整数, 则有下面的

引理 3 如果 $\operatorname{gcd}\left(k, q^{2}\right)=q_{1}{ }^{i_{1}} q_{2}{ }^{i_{2}} \cdots q_{\alpha}{ }^{i_{\alpha}} q_{\alpha+1}{ }^{2 k_{\alpha+1}-i_{\alpha+1}} p_{\alpha+2}{ }^{2 k_{\alpha+2}-i_{\alpha+2}} \cdots$ $p_{\beta}{ }^{2 k_{\beta}-i_{\beta}} q_{\beta+1}{ }^{2 k_{\beta+1}} q_{\beta+2}{ }^{2 k_{\beta+2}} \cdots q_{\gamma}{ }^{2 k_{\gamma}}$, 其中 $1 \leqslant \alpha \leqslant \beta \leqslant \gamma, 0 \leqslant i_{j} \leqslant k_{j}$ (当 $1 \leqslant j \leqslant$ $\alpha$ 时) 或 $0<i_{j}<k_{j}$ (当 $\alpha+1 \leqslant j \leqslant \beta$ 时); $\operatorname{gcd}\left(e, p^{2}\right)=p_{1}{ }^{j_{1}} p_{2}{ }^{j_{2}} \cdots p_{\mu}{ }^{j_{\mu}} p_{\mu+1}{ }^{2 h_{\mu+1}-j_{\mu+1}}$ $p_{\mu+2}{ }^{2 h_{\mu+2}-j_{\mu+2}} \cdots p_{\nu}{ }^{2 h_{\nu}-j_{\nu}} p_{\nu+1}{ }^{2 h_{\nu+1}} p_{\nu+2}{ }^{2 h_{\nu+2}} \cdots p_{\omega}{ }^{2 h_{\omega}}$, 其中 $1 \leqslant \mu \leqslant \nu \leqslant \omega, 0 \leqslant$ $j_{i} \leqslant h_{i}$ (当 $1 \leqslant i \leqslant \mu$ 时) 或 $0<j_{i}<h_{i}$ (当 $\mu+1 \leqslant i \leqslant \nu$ 时), 设

$$
\begin{aligned}
& s=q_{1}{ }^{i_{1}} q_{2}{ }^{i_{2}} \cdots q_{\alpha}{ }^{i_{\alpha}}, t=q_{1}{ }^{k_{1}-i_{1}} q_{2}{ }^{k_{2}-i_{2}} \cdots q_{\alpha}{ }^{k_{\alpha}-i_{\alpha}}, u=q_{\alpha+1}{ }^{i_{\alpha+1}} q_{\alpha+2}{ }^{i_{\alpha+2}} \cdots q_{\beta}{ }^{i_{\beta}} \text {, } \\
& v=q_{\alpha+1}{ }^{k_{\alpha+1}-i_{\alpha+1}} q_{\alpha+2}{ }^{k_{\alpha+2}-i_{\alpha+2}} \cdots q_{\beta}^{k_{\beta}-i_{\beta}}, w=q_{\beta+1}{ }^{k_{\beta+1}} q_{\beta+2}{ }^{k_{\beta+2}} \cdots q_{\gamma}^{k_{\gamma}}, \\
& s^{\prime}=p_{1}^{j_{1}} p_{2}^{j_{2}} \cdots p_{\mu}{ }^{j_{\mu}}, t^{\prime}=p_{1}^{h_{1}-j_{1}}{p_{2}}^{h_{2}-j_{2}} \cdots p_{\mu}{ }^{h_{\mu}-j_{\mu}}, u^{\prime}=p_{\mu+1}{ }^{j_{\mu+1}} p_{\mu+2}{ }^{j_{\mu+2}} \cdots p_{\nu}{ }^{j_{\nu}}, \\
& v^{\prime}=p_{\mu+1}^{h_{\mu+1}-j_{\mu+1}} p_{\mu+2}{ }^{h_{\mu+2}-j_{\mu+2}} \cdots p_{\nu}^{h_{\nu}-j_{\nu}}, w^{\prime}=p_{\nu+1}^{h_{\nu+1}} p_{\nu+2}{ }^{h_{\nu+2}} \cdots p_{\omega}^{h_{\omega}},
\end{aligned}
$$

并设 $k=s u v^{2} w^{2} k^{\prime}, e=s^{\prime} u^{\prime} v^{\prime 2} w^{\prime 2} e^{\prime}$, 则

$$
\begin{gathered}
m=s t u s^{\prime} t^{\prime} u^{\prime}\left(s^{\prime} u^{\prime} v^{2} w^{\prime 2} e^{\prime}+s u v^{2} w^{2} k^{\prime}\right)\left(t v^{\prime} w^{\prime} e^{\prime}+v w t^{\prime} k^{\prime}\right) z^{\prime}, \\
n=s u v w s^{\prime} u^{\prime} v^{\prime} w^{\prime}\left(s t^{2} u e^{\prime}+s^{\prime} t^{\prime 2} u^{\prime} k^{\prime}\right)\left(t v^{\prime} w^{\prime} e^{\prime}+v w t^{\prime} k^{\prime}\right) z^{\prime}, \\
a=s t u s^{\prime} u^{\prime} v^{\prime} w^{\prime} e^{\prime}\left(t v^{\prime} w^{\prime} e^{\prime}+v w t^{\prime} k^{\prime}\right) z^{\prime}, \quad b=s u v w s^{\prime} t^{\prime} u^{\prime} k^{\prime}\left(t v^{\prime} w^{\prime} e^{\prime}+v w t^{\prime} k^{\prime}\right) z^{\prime}, \\
r=\left(s^{\prime} u^{\prime} v^{\prime 2} w^{\prime 2} e^{\prime}+s u v^{2} w^{2} k^{\prime}\right)\left(s t^{2} u e^{\prime}+s^{\prime} t^{\prime 2} u^{\prime} k^{\prime}\right) z^{\prime}, d=s t u s^{\prime} t^{\prime} u^{\prime}\left(t v^{\prime} w^{\prime} e^{\prime}+v w t^{\prime} k^{\prime}\right) z^{\prime},
\end{gathered}
$$

其中 $z^{\prime}$ 是正整数.

证 由条件有 $\operatorname{gcd}\left(k, q^{2}\right)=s u v^{2} w^{2}, \operatorname{gcd}\left(e, p^{2}\right)=s^{\prime} u^{\prime} v^{\prime 2}{w^{\prime}}^{2}$ 和 $k=s u v^{2} w^{2} k^{\prime}$, $e=s^{\prime} u^{\prime} v^{\prime 2}{w^{\prime}}^{2} e^{\prime}$. 因为 $\operatorname{gcd}(e, k)=1$, 故又有 $\operatorname{gcd}\left(e^{\prime}, k^{\prime}\right)=1, \operatorname{gcd}\left(s^{\prime} u^{\prime} v^{\prime 2}{w^{\prime}}^{2} e^{\prime}\right.$, $\left.s u v^{2} w^{2} k^{\prime}\right)=1$. 于是由引理 1 , 有 $\operatorname{gcd}\left(e^{\prime} k^{\prime}, s^{\prime} u^{\prime} v^{\prime 2} w^{\prime 2} e^{\prime}+s u v^{2} w^{2} k^{\prime}\right)=1$. 令 $\operatorname{gcd}\left(s t^{2} u, s^{\prime} t^{\prime 2} u^{\prime}\right)=\xi$, 则

$$
\operatorname{gcd}\left(\frac{s t^{2} u e^{\prime}}{\xi}, \frac{s^{\prime} t^{\prime 2} u^{\prime} k^{\prime}}{\xi}\right)=1 .
$$

由引理 1 可得

$$
\operatorname{gcd}\left(e^{\prime} k^{\prime}, \frac{s t^{2} u e^{\prime}}{\xi}+\frac{s^{\prime} t^{\prime 2} u^{\prime} k^{\prime}}{\xi}\right)=1
$$

www.scichina.com 
又因为 $\operatorname{gcd}\left(e^{\prime} k^{\prime}, \xi\right)=1$, 所以有 $\operatorname{gcd}\left(e^{\prime} k^{\prime}, s t^{2} u e^{\prime}+s^{\prime} t^{\prime 2} u^{\prime} k^{\prime}\right)=1$. 由于

$$
r=\frac{\left(s^{\prime} u^{\prime} v^{\prime 2} w^{\prime 2} e^{\prime}+s u v^{2} w^{2} k^{\prime}\right)\left(s t^{2} u e^{\prime}+s^{\prime} t^{\prime 2} u^{\prime} k^{\prime}\right) z}{e^{\prime} k^{\prime}}
$$

是正整数, 因而 $\frac{z}{e^{\prime} k^{\prime}}$ 一定是正整数. 令 $z^{\prime}=\frac{z}{e^{\prime} k^{\prime}}$, 即得引理中的各式.

为了证明定理 3 , 由引理 2 , 只需要下面的直接构作:

引理 4 对于任意正整数 $s, t, u, v, w, s^{\prime}, t^{\prime}, u^{\prime}, v^{\prime}, w^{\prime}, e$ 和 $k$, 如果

$$
\begin{gathered}
m=s t u s^{\prime} t^{\prime} u^{\prime}\left(s^{\prime} u^{\prime} v^{\prime 2} w^{2} e+s u v^{2} w^{2} k\right)\left(t v^{\prime} w^{\prime} e+v w t^{\prime} k\right), \\
n=s u v w s^{\prime} u^{\prime} v^{\prime} w^{\prime}\left(s t^{2} u e+s^{\prime} t^{2} u^{\prime} k\right)\left(t v^{\prime} w^{\prime} e+v w t^{\prime} k\right),
\end{gathered}
$$

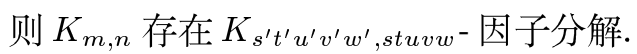

证 记 $a=s t u s^{\prime} u^{\prime} v^{\prime} w^{\prime} e\left(t v^{\prime} w^{\prime} e+v w t^{\prime} k\right), b=s u v w s^{\prime} t^{\prime} u^{\prime} k\left(t v^{\prime} w^{\prime} e+v w t^{\prime} k\right)$, $r=\left(s^{\prime} u^{\prime} v^{\prime 2} w^{\prime 2} e+s u v^{2} w^{2} k\right)\left(s t^{2} u e+s^{\prime} t^{\prime 2} u^{\prime} k\right), r_{1}=s^{\prime} u^{\prime} v^{\prime 2}{w^{\prime}}^{2} e+s u v^{2} w^{2} k$ 和 $r_{2}=$ $s t^{2} u e+s^{\prime} t^{\prime 2} u^{\prime} k$, 并令 $X$ 和 $Y$ 是 $K_{m, n}$ 的两个部分点集,

$$
\begin{gathered}
X=\left\{x_{i, j}: 1 \leqslant i \leqslant r_{1} ; 1 \leqslant j \leqslant s t u s^{\prime} t^{\prime} u^{\prime}\left(t v^{\prime} w^{\prime} e+v w t^{\prime} k\right)\right\}, \\
Y=\left\{y_{i, j}: 1 \leqslant i \leqslant r_{2} ; 1 \leqslant j \leqslant s u v w s^{\prime} u^{\prime} v^{\prime} w^{\prime}\left(t v^{\prime} w^{\prime} e+v w t^{\prime} k\right)\right\} .
\end{gathered}
$$

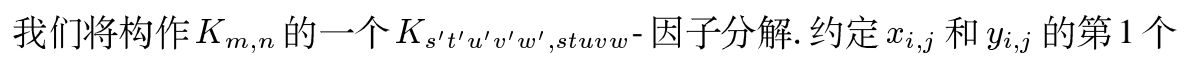
下标分别在 $\left\{1,2, \cdots, r_{1}\right\}$ 和 $\left\{1,2, \cdots, r_{2}\right\}$ 中进行模 $r_{1}$ 和 $r_{2}$ 的运算, 它们的第 2 个 下标分别在 $\left\{1,2, \cdots, s t u s^{\prime} t^{\prime} u^{\prime}\left(t v^{\prime} w^{\prime} e+v w t^{\prime} k\right)\right\}$ 和 $\left\{1,2, \cdots, s u v w s^{\prime} u^{\prime} v^{\prime} w^{\prime}\left(t v^{\prime} w^{\prime} e+\right.\right.$ $\left.\left.v w t^{\prime} k\right)\right\}$ 中进行模 $s t u s^{\prime} t^{\prime} u^{\prime}\left(t v^{\prime} w^{\prime} e+v w t^{\prime} k\right)$ 和 $s u v w s^{\prime} u^{\prime} v^{\prime} w^{\prime}\left(t v^{\prime} w^{\prime} e+v w t^{\prime} k\right)$ 的运 算.

对于每一个 $i, x, x^{\prime}, y, y^{\prime}, z$ 和 $z^{\prime}, 1 \leqslant i \leqslant e, 1 \leqslant x \leqslant v w, 1 \leqslant x^{\prime} \leqslant v^{\prime} w^{\prime}, 1 \leqslant$ $y \leqslant s t u, 1 \leqslant y^{\prime} \leqslant s^{\prime} t^{\prime} u^{\prime}, 1 \leqslant z \leqslant t$ 和 $1 \leqslant z^{\prime} \leqslant s^{\prime} u^{\prime} v^{\prime} w^{\prime}$, 令 $f\left(z, y^{\prime}\right)=s u\left(t v^{\prime} w^{\prime} e+\right.$ $\left.v w t^{\prime} k\right)\left(t y^{\prime}+z-t-1\right), g(i, y, z)=s t^{2} u(i-1)+t(y-1)+z$ 和 $h\left(i, x, x^{\prime}, y, z^{\prime}\right)=$ $s t u(i-1)+s t u\left(x^{\prime}-1\right) e+s u\left(t v^{\prime} w^{\prime} e+v w t^{\prime} k\right)\left(v w z^{\prime}+x-v w-1\right)+y-1$, 且构作 如下边集:

$$
\begin{aligned}
E_{i}= & \left\{x_{i+\left(z^{\prime}-1\right) e+s^{\prime} u^{\prime} v^{\prime} w^{\prime}\left(x^{\prime}-1\right) e, f\left(z, y^{\prime}\right)+j} y_{g(i, y, z), h\left(i, x, x^{\prime}, y, z^{\prime}\right)+j}:\right. \\
& 1 \leqslant j \leqslant s u\left(t v^{\prime} w^{\prime} e+v w t^{\prime} k\right), 1 \leqslant x \leqslant v w, 1 \leqslant x^{\prime} \leqslant v^{\prime} w^{\prime}, \\
& \left.1 \leqslant y \leqslant s t u, 1 \leqslant y^{\prime} \leqslant s^{\prime} t^{\prime} u^{\prime}, 1 \leqslant z \leqslant t, 1 \leqslant z^{\prime} \leqslant s^{\prime} u^{\prime} v^{\prime} w^{\prime}\right\} .
\end{aligned}
$$

对于每一个 $i, x, x^{\prime}, y, y^{\prime}$ 和 $z, 1 \leqslant i \leqslant k, 1 \leqslant x \leqslant s u v w, 1 \leqslant x^{\prime} \leqslant s^{\prime} u^{\prime} v^{\prime} w^{\prime}, 1 \leqslant$ $y \leqslant v w, 1 \leqslant y^{\prime} \leqslant s^{\prime} t^{\prime} u^{\prime}$ 和 $1 \leqslant z \leqslant t^{\prime}$, 令 $\varphi(i, x, y)=s^{\prime} u^{\prime} v^{\prime 2} w^{\prime 2} e+s u v^{2} w^{2}(i-1)+$ $v w(x-1)+y$ 和 $\psi\left(i, x, x^{\prime}, y, z\right)=s t u v^{\prime} w^{\prime} e+s u\left(t v^{\prime} w^{\prime} e+v w t^{\prime} k\right)\left(v w x^{\prime}+y-v w-\right.$ 1) $+\operatorname{suvw}(i+z k-k-1)+x-1$, 且构作边集

$$
\begin{aligned}
E_{e+i}= & \left\{x_{\varphi(i, x, y), s t u\left(t v^{\prime} w^{\prime} e+v w t^{\prime} k\right)\left(y^{\prime}-1\right)+j} y_{s t^{2} u e+\left(t^{\prime} y^{\prime}+z-t^{\prime}-1\right) k+i, \psi\left(i, x, x^{\prime}, y, z\right)+j}:\right. \\
& 1 \leqslant j \leqslant s t u\left(t v^{\prime} w^{\prime} e+v w t^{\prime} k\right), 1 \leqslant x \leqslant s u v w, 1 \leqslant x^{\prime} \leqslant s^{\prime} u^{\prime} v^{\prime} w^{\prime}, \\
& \left.1 \leqslant y \leqslant v w, 1 \leqslant y^{\prime} \leqslant s^{\prime} t^{\prime} u^{\prime}, 1 \leqslant z \leqslant t^{\prime}\right\} .
\end{aligned}
$$


令 $F=\cup_{1 \leqslant i \leqslant e+k} E_{i}$, 则图 $F$ 就是 $K_{m, n}$ 的一个 $K_{s^{\prime} t^{\prime} u^{\prime} v^{\prime} w^{\prime}, s t u v w^{-}}$因子. 定 义 $X \cup Y$ 到 $X \cup Y$ 上的双射 $\sigma: \sigma\left(x_{i, j}\right)=x_{i+1, j}, \sigma\left(y_{i, j}\right)=y_{i+1, j}$. 对于每一个 $i \in\left\{1,2, \cdots, r_{1}\right\}$ 和每一个 $j \in\left\{1,2, \cdots, r_{2}\right\}$, 令

$$
F_{i, j}=\left\{\sigma^{i}(x) \sigma^{j}(y) \mid x \in X, y \in Y, x y \in F\right\} .
$$

易证每一个图 $F_{i, j}\left(1 \leqslant i \leqslant r_{1}, 1 \leqslant j \leqslant r_{2}\right)$ 都是 $K_{m, n}$ 的 $K_{s^{\prime} t^{\prime} u^{\prime} v^{\prime} w^{\prime}, s t u v w}$ - 因子. 由于它们边集的并构成 $K_{m, n}$, 因此 $\left\{F_{i, j} \mid 1 \leqslant i \leqslant r_{1}, 1 \leqslant j \leqslant r_{2}\right\}$ 就是 $K_{m, n}$ 的一 个 $K_{s^{\prime} t^{\prime} u^{\prime} v^{\prime} w^{\prime}, s t u v w}$ - 因子分解. 引理得证.

定理 3 的证 由引理 2 4 知, 对于满足定理 3 条件的 $m$ 和 $n, K_{m, n}$ 存在 $K_{p, q^{-}}$因子分解.

\section{2 定理 4 的证明}

首先证明两个引理.

引理 5 对于任意正整数 $s$, 如果 $K_{m, n}$ 存在 $K_{p, q}$ - 因子分解, 则 $K_{m s, n s}$ 存在 $K_{p s, q s^{-}}$因子分解.

证 设 $\left\{F_{i}: 1 \leqslant i \leqslant r\right\}$ 是 $K_{m, n}$ 的一个 $K_{p, q^{-}}$因子分解. 对于每一个 $i \in\{1,2, \cdots, r\}$, 用 $s$ 个点代替 $F_{i}$ 的每个点, 用 $K_{s, s}$ 代替 $F_{i}$ 的每条边, 可得到 $K_{m s, n s}$ 的一个支撑子图 $G_{i}$, 且 $G_{i}(1 \leqslant i \leqslant r)$ 边集的并为 $K_{m s, n s}$. 因为 $K_{m, n}$ 是 可 $K_{p, q}$ - 因子分解的, 所以 $G_{i}$ 是可 $K_{p, q}$ 因子分解的, 故 $K_{m s, n s}$ 存在 $K_{p s, q s}$ - 因 子分解.

引理 6 对于任意正整数 $s$, 如果 $m$ 和 $n$ 满足 $p=p_{0} s, q=q_{0} s$ 情形时的 BAC 条件, 则存在正整数 $m_{0}$ 和 $n_{0}$ 满足 $m=m_{0} s, n=n_{0} s$ 且适合 $p=p_{0}, q=q_{0}$ 情形 时的 BAC 条件.

证 由条件知 $m$ 和 $n$ 满足 $p=p_{0} s, q=q_{0} s$ 情形时的 BAC 条件, 于是知

$$
\frac{p_{0} s n-q_{0} s m}{\left(p_{0}^{2}-q_{0}^{2}\right) s^{2}}=\frac{\frac{p_{0}(m+n)}{\left(p_{0}+q_{0}\right) s}-\frac{m}{s}}{p_{0}-q_{0}}, \frac{p_{0} s m-q_{0} s n}{\left(p_{0}^{2}-q_{0}^{2}\right) s^{2}}=\frac{\frac{p_{0}(m+n)}{\left(p_{0}+q_{0}\right) s}-\frac{n}{s}}{p_{0}-q_{0}}, \frac{m+n}{\left(p_{0}+q_{0}\right) s}
$$

是整数. 因而 $\frac{m}{s}, \frac{n}{s}$ 是整数. 令 $m_{0}=\frac{m}{s}, n_{0}=\frac{n}{s}$. 容易验证正整数 $m_{0}$ 和 $n_{0}$ 适 合 $p=p_{0}, q=q_{0}$ 情形时的 BAC 条件. 引理得证.

当 $p=k, q=k+1$ 时, $K_{m, n}$ 的 $K_{k, k+1}$ - 因子分解问题的 BAC 条件为, 下列 各式都是整数:

$$
\begin{aligned}
\frac{m+n}{2 k+1}, & \frac{(k+1) m-k n}{2 k+1}, \frac{(k+1) n-k m}{2 k+1}, \frac{(2 k+1) m n}{k(k+1)(m+n)}, \\
\frac{((k+1) n-k m) n}{(k+1)(m+n)}, & \frac{((k+1) m-k n) n}{k(m+n)}, \frac{((k+1) m-k n) m}{(k+1)(m+n)}, \frac{((k+1) n-k m) m}{k(m+n)} .
\end{aligned}
$$

由

$$
\frac{(k+1) m-k n}{2 k+1}, \frac{(k+1) n-k m}{2 k+1}
$$

是整数, 可得到定理 3 的条件 (1) $k n \leqslant(k+1) m,(2) k m \leqslant(k+1) n$ 和 (3) $(k+$ 
1) $m-k n \equiv(k+1) n-k m \equiv 0(\bmod (2 k+1))$. 再由

$$
\begin{aligned}
r=\frac{(2 k+1) m n}{k(k+1)(m+n)}= & \frac{(k+1) m-k n}{2 k+1}+\frac{(k+1) n-k m}{2 k+1} \\
& +\frac{[(k+1) n-k m][(k+1) m-k n]}{k(k+1)(2 k+1)(m+n)}
\end{aligned}
$$

是整数, 可得到定理 3 的条件 (4) $((k+1) m-k n)((k+1) n-k m) \equiv 0(\bmod k(k+$ $1)(2 k+1)(m+n))$. 根据定理 3, 当 $p=k, q=k+1$ 时, $K_{m, n}$ 存在 $K_{p, q^{-}}$因子分 解, 即 Martin 的 BAC 猜想成立.

引理 7 对于任意正整数 $k$, 当 $p=k, q=k+1$ 时, $\mathrm{BAC}$ 猜想成立.

定理 4 的证 结合引理 5 7, 知对于任意正整数 $k$, 当 $p: q=k:(k+1)$ 时,

BAC 猜想成立.

\section{参考文献}

1 Ushio K. G-designs and related designs. Discrete Math, 1993, 116: 299 311

2 Bondy J A, Murty U S R. Graph Theory with Applications. London: Macmillan Press, 1976

3 Yamamoto S, Tazawa S, Ushio K, et al. Design of a balanced multiple-valued file organization scheme with the least redundancy. ACM Trans Database Systems, 1979, 4: 518 530

4 Ushio K. $P_{3}$-factorization of complete bipartite graphs. Discrete Math, 1988, 72: 361 366

5 Wang H. On $K_{1, k}$-factorizations of a complete bipartite graph. Discrete Math, 1994, 126: 359 364

6 Du B L. $K_{1, p^{2}}$-factorization of complete bipartite graphs. Discrete Math, 1998, 187: 273 279

7 Du B L. $K_{1, p q}$-factorization of complete bipartite graphs. Austral J Combin, 2002, 26: 85 92

8 Du B L, Wang J. $K_{1, k}$-factorizations of complete bipartite graphs. Discrete Math, 2002, 259: 301 306

9 Martin N. Complete bipartite factorisations by complete bipartite graphs. Discrete Math, 1997, 167/168: $461 \sim 480$ 